\title{
HILBERT SPACE METHODS IN THE THEORY OF HARMONIC INTEGRALS $\left({ }^{1}\right)$
}

\author{
BY \\ MATTHEW P. GAFFNEY
}

The theory of harmonic integrals was created by Hodge [15], and the theorem which bears his name is the central result of the subject. Kodaira [17] and-independently-de Rham and Bidal [1] used the generalized harmonic operator $\Delta$ in their treatments of the theory. $\Delta$ was also used by Milgram and Rosenbloom [19] in their study of harmonic integrals with the heat equation. It is our purpose to develop the properties of $\Delta$ from the point of view of Hilbert space theory, thus arriving at Hodge's theorem without the use of a generalized integral equation theory. In addition, in $\$ 2$ we study $\Delta$ on a class of open manifolds-those with negligible boundary; these include all complete manifolds. $\$ 3$ contains a proof of the fundamental differentiability lemma.

We wish to express our appreciation to Professor M. H. Stone, who suggested this topic to us. He provided us with the proof (in $\S 2$ ) that $I+\bar{\delta} \delta^{*}+\bar{d} d^{*}$ is self-adjoint, and he suggested the application of Rellich's results to the proof of the complete continuity of the Green's operator.

1. Compact manifolds; Hodge's theorem. We assume familiarity with the basic concepts of differential forms on Riemannian manifolds. Expositions of this material are contained in [1], [4], [7], [13], and [15].

The index notation of Kodaira considerably simplifies some of the notational problems of tensor calculus. A capital letter is to denote a set of $p$ indices: $I=\left\langle i_{1}, \cdots, i_{p}\right\rangle$. When a capital letter appears where there is room for only one index a multiplication is implied. Thus $d x^{I}=d x^{i_{1}} \wedge \cdots \wedge d x^{i_{p}}$ and $g^{I, J}=g^{i_{1} j_{1}} \cdots g^{i_{p} i_{p}}$. For summations, we shall use $\sum$ (or $\sum_{I}$ ) to indicate summation over all permutations of the indices $p$ at a time, while $\sum^{\prime}$ indicates that from each combination only one permutation is used; we shall take the one in increasing order. $\sum$ or $\sum^{\prime}$ without a subscript applies only to indices which occur both above and below. In this notation a form $\alpha$ is written out as $\alpha=\sum^{\prime} A_{I} d x^{I}$; indices are raised by $T^{I}=\sum g^{I, J} T_{J}$.

In this section we consider a compact, oriented, Riemannian manifold $M$

Presented to the Society, November 24, 1950, under the title The harmonic operator for exterior differential forms; received by the editors January 9, 1951 and, in revised form, August 5, 1953.

(1) This paper was sponsored by the Office of Naval Research, under a contract at the University of Chicago and by the Office of Ordnance Research, U. S. Army under a contract at Princeton University. It is a substantial revision of parts of the author's dissertation [13], which was submitted in partial fulfilment of the requirements for the Ph.D. degree at the University of Chicago. The earlier work was reported in [12]. 
whose Riemann tensor is of class $C^{k}, k=5, \cdots, \infty$. (As a manifold $M$ must be $C^{k+1}$.) $\delta \equiv(-1)^{n p+n+1} * d *$ ( $p$ is the degree of the form to which $\delta$ is applied; $n$ the dimension of $M$ ), and $\Delta \equiv d \delta+\delta d$. (For functions $\Delta$ is the negative of the Laplace-Beltrami operator.) Inner products are defined by $(\alpha, \beta) \equiv \int \alpha * \beta$. A corollary of Stokes's theorem is that $d$ and $\delta$ are adjoint (cf. $\$ 2$ ). In fact, from the product formula for $d$ and the definition of $\delta$,

$$
d(\alpha * \beta)=d \alpha * \beta-\alpha * \delta \beta,
$$

where

$$
\alpha=\alpha^{p}, \quad \beta=\beta^{p+1} ;
$$

since by Stokes's theorem the integral of the left side is zero, one obtains $(d \alpha, \beta)=(\alpha, \delta \beta)$.

Let $F$ be the class of harmonic forms-those forms which $\Delta$ maps into 0 . Since $(\Delta \alpha, \alpha)=(d \alpha, d \alpha)+(\delta \alpha, \delta \alpha)$, one sees that harmonicity is equivalent to $d \alpha=0$ and $\delta \alpha=0$. Therefore $F$ is orthogonal to both $R(d)$ (= range of $d$ ) and $R(\delta)$.

$R(d)$ and $R(\delta)$ are themselves orthogonal, for consider $(d \alpha, \delta \beta)$ : if either $\alpha$ or $\beta$ (say $\alpha)$ is $C^{2}$, then $(d \alpha, \delta \beta)=(d d \alpha, \beta)=0$. Even when $\alpha$ and $\beta$ are both $C^{1}$ it is true that $(d \alpha, \delta \beta)=0$; this can be demonstrated with the aid of a good smoothing operator such as the Friedrichs mollifier. An alternative would be to restrict the domains of $d$ and $\delta$ to $C^{2}$ forms; the domain of $\Delta$ must then be correspondingly restricted.

We follow Bidal and de Rham [1] in noting that the uniqueness assertion of de Rham's theorem together with the adjointness of $d$ and $\delta$ already implies the uniqueness of harmonic integrals with prescribed periods. (The period of a $p$-form on a $p$-cycle is the value of the integral of the form over the cycle.) For if $\alpha$ is harmonic with zero periods, then by de Rham's theorem $\alpha=d \theta$. But then $(\alpha, \alpha)=(\alpha, d \theta)=(\delta \alpha, \theta)=(0, \theta)=0$, so $\alpha$ is zero.

The Hilbert space $H^{p}$ is the class of all measurable $p$-forms $\alpha$ such that $(\alpha, \alpha)$ is finite, provided with the above inner product and the usual equivalence relation (compare the definition in [13]). $\Delta$ is a densely defined linear operator on $H^{p}$ and because of the adjointness of $d$ and $\delta$ it is symmetric $[(\Delta \alpha, \beta)=(\alpha, \Delta \beta)]$. (The $C^{k}$ forms are dense in $H^{p}$ even when $M$ is open. One way of establishing the density is to use a smoothing operator. See [12] and [13].)

Following Kodaira [18, p. 605] we establish the weak decomposition formula:

$$
H^{p}=\bar{R}\left(d^{p-1}\right)+\bar{R}\left(\delta^{p+1}\right)+F^{p} .
$$

The bar indicates closure; we are able to leave it off $F^{p}$ since if a sequence of harmonic forms $\alpha_{i}$ approaches $\alpha$ (in the Hilbert space norm), then 


$$
(\Delta \theta, \alpha)=\lim \left(\Delta \theta, \alpha_{i}\right)=\lim \left(\theta, \Delta \alpha_{i}\right)=0
$$

for all $\theta$ of class $C^{2}$. By the fundamental differentiability lemma ( $\left.\$ 3\right) \alpha$ belongs to $F^{p}$.

To prove (1) one need only establish that $R(\Delta)$ is dense in $\left(F^{p}\right)^{\perp}$, the set of all elements orthogonal to the harmonic forms. Suppose that $\gamma$ is orthogonal to the range of $\Delta$, so that $(\Delta \theta, \gamma)=0$ for all $\theta$ of class $C^{2}$. It has just been remarked that $(\Delta \theta, \gamma)=0$ implies $\gamma$ belongs to $F^{p}$. This means that no nonzero form in $\left(F^{p}\right)^{\perp}$ is orthogonal to $R(\Delta)$, nor to the larger set $R\left(d^{p-1}\right)+R\left(\delta^{p+1}\right)$; therefore no nonzero element is orthogonal to the closed linear manifold given by the right-hand side of (1). But in a Hilbert space a closed linear manifold to which no non-zero element is orthogonal is the whole space.

It is possible [18] to obtain Hodge's existence theorem from (1) and de Rham's theorem. We shall wait until we have the full decomposition theorem, from which it follows more easily. (The complete continuity of $G$ will be used to obtain the full theorem.)

We wish to prove that $\bar{\Delta}$, the closure of $\Delta$, is self-adjoint. (The closure of $\Delta$ is defined as follows: take the graph of $\Delta$ in $H^{p} \times H^{p}$; close it. This closure is the graph of $\bar{\Delta}$. "Self-adjoint" means: equals its maximal adjoint operator.)

Let $\Delta_{1}=\Delta+I$. Since

$$
\overline{\Delta+I}=\bar{\Delta}+I,
$$

the notation $\bar{\Delta}_{1}$ is unambiguous. The self-adjointness of $\bar{\Delta}$ is equivalent to that of $\bar{\Delta}_{1}$, and it is the latter we examine. $\Delta_{1}$ is semibounded in accord with the inequality

$$
\left(\Delta_{1} \alpha, \alpha\right)=(d \alpha, d \alpha)+(\delta \alpha, \delta \alpha)+(\alpha, \alpha) \geqq(\alpha, \alpha) .
$$

From the inequality it is seen that $\Delta_{1}$ is one-to-one. Furthermore, the mapping in the other direction $\left(\Delta_{1}^{-1}\right)$ may not (a priori) be densely defined, but at least it is bounded, since (from the semiboundedness inequality)

$$
\left.\|\alpha\|^{2} \leqq\left\|\Delta_{1} \alpha\right\|\|\alpha\| \quad \text { (or }\|\alpha\| \leqq\left\|\Delta_{1} \alpha\right\|\right) \text {. }
$$

(The norm of $\alpha$ is given by $\|\alpha\|=(\alpha, \alpha)^{1 / 2}$.) Therefore $\bar{\Delta}_{1}$ maps onto a closed subset of $H^{p}$. The only way this subset could fail to be all of $H^{p}$ is that there exist a nonzero form $\alpha$ orthogonal to the range of $\Delta_{1}$, that is, $\left(\Delta_{1} \theta, \alpha\right)=0$ for all $C^{2}$ forms $\theta$. But by the fundamental differentiability lemma ( $\$ 3$; take $\lambda=-1)$ this would imply that $\alpha$ is $C^{2}$ and $\Delta_{1} \alpha=0$. Since $\Delta_{1}$ is one-to-one $\alpha$ must be zero, so $\bar{\Delta}_{1}$ maps onto $H^{p}$ itself. Therefore $\left(\bar{\Delta}_{1}\right)^{-1}$ is a bounded, symmetric operator defined on all of $H^{p}$ and must be self-adjoint, forcing its inverse $\bar{\Delta}_{1}$ to be so too. From this we obtain the

Proposition. On a compact, orientable, Riemannian manifold $\bar{\Delta}$ is selfadjoint. 
We proceed to develop an inequality which will be useful in establishing the complete continuity of $\left(\bar{\Delta}_{1}\right)^{-1}$. Consider $C^{2}$ forms $\alpha$ vanishing outside a coordinate neighborhood $K$; and define the Dirichlet integral

$$
D(\alpha)=\int \sum_{I, k}^{\prime}\left(\frac{\partial A_{I}(x)}{\partial x^{k}}\right)^{2} d x .
$$

In euclidean space, where $\Delta$ acts componentwise,

$$
(\Delta \alpha, \alpha)=-\int \sum_{I, k}^{\prime}\left[\frac{\partial^{2}}{\left(\partial x^{b}\right)^{2}} A_{I}(x)\right] A_{I}(x) d x ;
$$

integrating the right side by parts we find that (in euclidean space)

$$
D(\alpha)=(\Delta \alpha, \alpha)=(d \alpha, d \alpha)+(\delta \alpha, \delta \alpha)
$$

(cf. Friedrichs [11], where the idea for the inequality was obtained).

In the general case it will be proved that:

Every point $P$ of $M$ is contained in a coordinate neighborhood $K$ such that for all $C^{2}$ forms $\alpha$ vanishing outside $K$

$$
D(\alpha) \leqq C[(d \alpha, d \alpha)+(\delta \alpha, \delta \alpha)+(\alpha, \alpha)]
$$

where $C$ is a constant depending on $K$.

Select a coordinate system in the neighborhood $K_{1}$ of $P$ with the property that $g_{i j}(P)=\delta_{i}^{j}$ (see appendix). Introduce a new euclidean tensor in $K_{1}$ by defining its components in this coordinate system to be $g_{i j}^{\prime}=\delta_{k}^{j}$. We shall use a prime to indicate that a quantity has been computed with respect to this tensor, as $\|\alpha\|^{\prime}$. As with any two Riemann tensors, we have $\|\alpha\|^{\prime 2} \leqq C_{1}\|\alpha\|^{2}$, etc. Thus

$$
\begin{aligned}
D(\alpha) & =\|d \alpha\|^{\prime 2}+\left\|\delta^{\prime} \alpha\right\|^{\prime 2} \leqq C_{1}\left[\|d \alpha\|^{2}+\left\|\delta^{\prime} \alpha\right\|^{2}\right] \\
& \leqq 2 C_{1}\left[\|d \alpha\|^{2}+\|\delta \alpha\|^{2}+\left\|\delta^{\prime} \alpha-\delta \alpha\right\|^{2}\right] .
\end{aligned}
$$

We need to know that

$$
\left\|\delta^{\prime} \alpha-\delta \alpha\right\|^{2} \leqq C_{2}\|\alpha\|^{2}+\epsilon(K) D(\alpha),
$$

where $\epsilon(K)$ approaches zero as the neighborhood $K$ shrinks to the point $P$. It suffices to prove the corresponding inequality for a typical term from a component of $\delta^{\prime} \alpha-\delta \alpha$,

$$
\left(H^{\prime} \frac{\partial}{\partial x^{i}} G^{\prime}-H \frac{\partial}{\partial x^{i}} G\right) A_{I}+\left(H^{\prime} G^{\prime}-H G\right) \frac{\partial}{\partial x^{i}} A_{I} .
$$

Let $\epsilon_{1}(K)$ be the upper bound of $\left|H^{\prime} G^{\prime}-H G\right|$ on $K$. Since the continuous function $H^{\prime} G^{\prime}-H G$ is zero at $P$ one sees that $\epsilon_{1}(K)$ does approach zero. Continuing, one obtains the above inequality for $\left\|\delta^{\prime} \alpha-\delta \alpha\right\|^{2}$ and therefore has 


$$
D(\alpha) \leqq 2 C_{1}\left[\|d \alpha\|^{2}+\|\delta \alpha\|^{2}+C_{2}\|\alpha\|^{2}+\epsilon(K) D(\alpha)\right] .
$$

Take $K$ so small that $2 C_{1} \epsilon(K)<1 / 2$, thus obtaining (2).

With the aid of (2) we shall give a direct proof that $\left(\bar{\Delta}_{1}\right)^{-1}$ is completely continuous, and from this obtain the complete continuity of the Green's operator $\left({ }^{2}\right)$.

We must prove that if a sequence of forms $\left\langle\alpha_{i}\right\rangle$ has the property that $\left\langle\left(\bar{\Delta}_{1}\right) \alpha_{i}\right\rangle$ is defined and bounded in norm, then a subsequence of $\left\langle\alpha_{i}\right\rangle$ converges (in the Hilbert space norm). It will suffice to consider the case in which the forms $\alpha_{i}$ are in the domain of $\Delta_{1}$.

From the hypothesis and the relation

$$
\left(\Delta_{1} \alpha, \Delta_{1} \alpha\right)=(\Delta \alpha, \Delta \alpha)+2(d \alpha, d \alpha)+2(\delta \alpha, \delta \alpha)+(\alpha, \alpha)
$$

it follows that $\left\langle\alpha_{i}\right\rangle,\left\langle d \alpha_{i}\right\rangle$, and $\left\langle\delta \alpha_{i}\right\rangle$ are bounded. The latter statement will still be true if we replace $\alpha_{i}$ by $\phi \alpha_{i}$, where $\phi$ is a component of a partition of unity. (We shall take the partition so fine that our Dirichlet integral inequality is valid.) To see that $\left\langle\delta \phi \alpha_{i}\right\rangle$ is bounded consider separately $\left\langle\phi \delta \alpha_{i}\right\rangle$, which clearly is bounded, and $*\left[d \phi * \alpha_{i}\right]$, which satisfies $\left\|*\left[d \phi * \alpha_{i}\right]\right\|$ $\leqq B\left\|\alpha_{i}\right\|$.

The individual components of $\left\langle\phi \alpha_{i}\right\rangle$ are bounded in norm, and so also are their first partial derivatives, as the Dirichlet integral inequality shows. But the hypothesis of the Rellich selection theorem [21] is just that a sequence $\left\langle f_{i}\right\rangle$ of functions along with the first derivatives is bounded in norm; the conclusion is that a convergent subsequence $\left\langle f_{i_{t}}\right\rangle$ can be selected. From here we easily backtrack to get the complete continuity of $\left(\bar{\Delta}_{1}\right)^{-1}$.

We give Rellich's proof (for cubic domains). The proof is based on the inequality

$$
\int f^{2} d x \leqq \frac{1}{s^{n}}\left[\int f d x\right]^{2}+\frac{n}{2} s^{2} \int \sum_{j}\left[\partial f / \partial x^{j}\right]^{2} d x
$$

where $f$ is an arbitrary $C^{1}$ function on some cube of width $s$, and the integration is taken over the cube.

To prove (3) observe that

$$
\begin{aligned}
{[f(x)-f(y)]^{2}=} & {\left[\int_{y^{1}}^{x^{1}} f_{1}\left(\xi^{1}, x^{2}, \cdots, x^{n}\right) d \xi^{1}+\int_{y^{2}}^{x^{2}} f_{2}\left(y^{1}, \xi^{2}, x^{3}, \cdots, x^{n}\right) d \xi^{2}\right.} \\
& \left.+\cdots+\int_{y^{n}}^{x^{n}} f_{n}\left(y^{1}, \cdots, y^{n-1}, \xi^{n}\right) d \xi^{n}\right]^{2} \\
\leqq & n s \sum_{j=1}^{n} \int_{0}^{\infty} f_{j}^{2}\left(y^{1}, \cdots, y^{j-1}, \xi^{j}, x^{j+1}, \cdots, x^{n}\right) d \xi^{j} .
\end{aligned}
$$

(2) Added in proof. In a recent note [26] Yosida has obtained this same proof. 
Integrating, and changing the order on the right,

$$
\iint[f(x)-f(y)]^{2} d x d y \leqq n s \sum_{j=1}^{n} \int f_{j}^{2}(z) d z \cdot s^{n+1} .
$$

Since $\iint[f(x)-f(y)]^{2} d x d y=2 \int f^{2}(z) d z \cdot s^{n}-2\left[\int f(z) d z\right]^{2}$, we have (3).

To continue with Rellich's proof, consider a cube $K$ of width $a$, which we cover with a sequence of nets. Let the $m$ th net be obtained by dividing each side of $K$ into $m$ equal segments, thus obtaining $m^{n}$ congruent cubes of width $s=a / m$. The step function obtained by replacing a function $g$ on each small cube of this net by its average value on that cube will be called the $m$ th approximation to $g$, denoted by $g^{m}$. (Thus for each $m$ there is a sequence of step functions $\left\langle f_{i}^{m}\right\rangle$.) On each small cube,

$$
\left|f_{i}^{m}\right|=\left|\int f_{i}\right| / s^{n} \leqq\left(\int f_{i}^{2} \cdot s^{n}\right)^{1 / 2} / s^{n}
$$

is bounded with respect to $i$. A bounded set of real numbers has an accumulation point, and therefore a subsequence of $\left\langle f_{i}^{m}\right\rangle$ converges on the small cube. By a limited diagonal process one obtains a subsequence which converges uniformly on the cube $K$.

The above result is true for each $m$. By a full diagonal process select $\left\langle i_{t}\right\rangle$ in such a way that for each $m$ the sequence $\left\langle f_{i_{t}}^{m}\right\rangle$ converges uniformly and therefore in the $L_{2}$ norm. We want to prove that $\left\langle f_{i_{i}}\right\rangle$ converges; this will be done if we show that $f_{i}^{m}$ approaches $f_{i}$ uniformly in $i$ as $m$ approaches $\infty$.

Consider $\int_{K}\left(f_{i}^{m}-f_{i}\right)^{2} d s$. Split the integration into integration over the small cubes of the net corresponding to $m$ and apply (1). Since the average value of $f_{i}^{m}-f_{i}$ is zero on each cube, (3) takes a simpler form; we obtain

$$
\int_{K}\left(f_{i}^{m}-f_{i}\right)^{2} d x \leqq \frac{n}{2}\left(\frac{a}{m}\right)^{2} \int\left(\sum_{i}\left(\frac{\partial f_{i}}{\partial x^{i}}\right)^{2} d x .\right.
$$

The right-hand integral is uniformly bounded, so the right side approaches zero uniformly in $i$ as $m$ approaches $\infty$. This proves the convergence and completes the proof.

Since the self-adjoint operator $\left(\bar{\Delta}_{1}\right)^{-1}$ is completely continuous, its spectrum consists of an infinity of characteristic values, each of finite multiplicity, which are bounded and have zero as their sole limit. The spectrum of $\bar{\Delta}_{1}$ again consists of characteristic values, which are respectively the reciprocals of those of $\left(\Delta_{1}\right)^{-1}$; in this correspondence multiplicity is preserved. The characteristic values of $\bar{\Delta}_{1}$ have no accumulation points and do not include zero. The spectrum of $\bar{\Delta}$ is obtained from that of $\bar{\Delta}_{1}$ by a translation of 1 unit to the left, with a preservation of multiplicities. Therefore the characteristic values of $\bar{\Delta}$ have no accumulation points. Furthermore, while zero may be a characteristic value, it has finite multiplicity, so we see that the 
number of linearly independent harmonic forms of degree $p$ is finite.

If we restrict $\bar{\Delta}$ to $F^{\perp}$, the orthogonal complement of the harmonic forms, it becomes one-to-one and has an inverse - it is this inverse which is understood by the notation $\bar{\Delta}^{-1}$. Either by the earlier proof or by the fact that an element orthogonal to the range of a self-adjoint operator is in its null space, the domain of $\bar{\Delta}^{-1}$ is dense in $F^{\perp}$. Since the characteristic values of the restricted $\bar{\Delta}$ have no accumulation point, $\bar{\Delta}^{-1}$ has a bounded spectrum (finite multiplicities) with 0 the sole accumulation point. $\bar{\Delta}^{-1}$ is therefore a completely continuous transformation. Being completely continuous it is bounded; it is therefore defined on all of $F^{\perp}$.

The Green's operator $G$, introduced by de Rham [6], is defined to be $\bar{\Delta}^{-1}$ on $F^{\perp}$ and 0 on $F$ itself. There follows immediately the

Proposition (DE Rham). The Green's operator $G$ on $M$ (compact) is completely continuous.

The fact that $\bar{\Delta}$ maps onto $F^{\perp}$, combined with the fundamental lemma, gives us

The full decomposition theorem (Bidal and de Rham). Any form $\psi$ of class $C^{1}$ can be written as a sum

$$
\psi=d(\delta \alpha)+\delta(d \alpha)+F(\psi),
$$

where $\alpha$ is of class $C^{2}$ and $F(\psi)$, the projection of $\psi$ on $F$, is of class $C^{k-4}$ (or $C^{2}$ if $k=5$ ). (If $\psi$ is $C^{m}, 1 \leqq m \leqq k-5$, then $\alpha$ is $C^{m+1}$.) Therefore $R(d)+R(\delta)+F$ is not only dense in $H^{p}$, but actually contains all $C^{1}$ forms.

Since $\psi-F(\psi)$ is in $F^{\perp}$ it is in the range of $\bar{\Delta}$, so there exists $\alpha$ with $\bar{\Delta} \alpha$ $=\psi-F(\psi)$. But $\psi-F(\psi)$ is of class $C^{m}$, and by the fundamental lemma $\alpha$ must be $C^{m+1}$ and hence in the domain of $\Delta$ itself. Therefore

$$
\psi-F(\psi)=\Delta \alpha=d(\delta \alpha)+\delta(d \alpha) .
$$

Bidal and de Rham [1] used this result to establish the existence part of

Hodge's THEOREM. Given a set of $\beta^{p}$ (pth Betti number) independent cycles on $M$ with real periods prescribed, there exists a unique harmonic p-form whose integral on each cycle gives the prescribed period $\left({ }^{3}\right)$.

The uniqueness has already been shown. By de Rham's theorem (a proof for small $k$ is given by Weil [22]) there exists a closed form $\psi$ of class $C^{1}$ which takes on the prescribed periods. Since $\psi$ is closed, it is orthogonal to the range of $\delta$ and in the decomposition we have only

$$
\psi=d(\delta \alpha)+F(\psi)
$$

(3) Added in proof. In [25] we obtain a different proof which does not use complete continuity. 
But by Stokes's theorem $d(\delta \alpha)$ integrates to zero on every cycle, so that $F(\psi)$ has the same periods as $\psi$, giving the existence.

de Rham [6] has shown that the full decomposition theorem will give the existence theorem directly without using de Rham's theorem. Hodge's [16] uniqueness proof is also independent of de Rham's theorem.

With $p=0$ (functions) Bochner [2] established the complete continuity of $\left(\Delta_{1}\right)^{-1}$ by the integral equation method as one of the steps in his proof that when $k=\omega$ (analyticity) $M$ can be analytically embedded in euclidean $(2 n+1)$-space.

2. Open manifolds. The assumption of compactness which was made on $M$ will now be dropped. In the study of differential operators on open manifolds we shall pay much closer attention to the domains of the operators involved; the definition of an operator on a Hilbert space must not only specify what is done, but also precisely which elements it is done to, and there is greater latitude in the selection of the domains of $d, \delta$, and $\Delta$ on open than on closed manifolds.

With $d$ and $\delta$ (but not $\Delta$ ) restricted to compact carried $C^{1}$ forms the weak decomposition theorem can be extended to the Hilbert space $H^{p}$ of measurable, square-integrable forms on $M$. (See Kodaira [18].)

For the remainder of this section we shall understand by $d$ the exterior differential operator with domain restricted to $C^{1}$ forms $\alpha$ such that both $\|\alpha\|$ and $\|d \alpha\|$ are finite; $\delta$ is similarly defined. According to standard Hilbert space conventions for combining operators (the domain of $S T$ consists of those $\sigma$ in the domain of $T$ such that $T \sigma$ is in the domain of $S$; the domain of $S+T$ is the intersection of the domains of $S$ and $T$ ), the domain of $\Delta$ is determined by those of $d$ and $\delta$.

Immediately the difficulty arises that with these domains $d$ and $\delta$ will not in general be adjoint and $\Delta$ will not be symmetric $\left({ }^{4}\right)$. The trouble is that the integral of the $n$-form $d(\alpha * \beta)$ (see $\$ 1$ ) is not necessarily zero; indeed, on the open unit sphere in euclidean $n$-space it is equal to the boundary integral of $\alpha * \beta$. We are therefore led to replace the missing compactness assumption with the assumption that $d$ and $\delta$ are adjoint (forcing $\Delta$ to be symmetric); we then say that the manifold has negligible boundary. Fortunately, a good many manifolds have this property; in particular, every complete Riemannian manifold has negligible boundary (see [14]). All the formal properties of orthogonality and symmetry which were stated in $\$ 1$ will be valid with the new assumption, which is maintained for the rest of this section. For example, since $(\Delta \alpha, \alpha)=(d \alpha, d \alpha)+(\delta \alpha, \delta \alpha)$, it follows that $\Delta \alpha=0$ if and only if $d \alpha=0$ and $\delta \alpha=0$. (The mere writing of $\Delta \alpha$ implies that $\alpha$ is in the domain of $\Delta$ as given above.) As in $\S 1$, one has the alternative of proving that $\bar{d}=\bar{d}_{2}$ (we shall assume this done) or dealing with $d_{2}$ and $\delta_{2}$ from the start. (The subscript indicates that the domain is restricted to $C^{2}$ forms, with the norm

(4) Added in proof. But see [25]. 
requirements unchanged.)

The adjoint $T^{*}$ of a densely defined operator $T$ on a Hilbert space is defined as follows: the domain of $T^{*}$ consists of all elements $\sigma$ such that there corresponds $\sigma^{*}$ with $(T \theta, \sigma)=\left(\theta, \sigma^{*}\right)$ for every $\theta$ in the domain of $T$; the value of $T^{*} \sigma$ is $\sigma^{*}$. According to a theorem of von Neumann (see Nagy [20, p. 30]), if $T$ is a densely defined closed linear transformation, then $T T^{*}$ and $T^{*} T$ are each self-adjoint. The method of proof is to show that $\left(I+T T^{*}\right)^{-1}$ is a bounded self-adjoint operator. Since the inverse of a self-adjoint operator is self-adjoint, this proves that $I+T T^{*}$ and therefore $T T^{*}$ are self-adjoint.

Thus $\bar{d} d^{*}$ and $\bar{\delta} \delta^{*}$ are self-adjoint. (They are extensions of $d \delta$ and $\delta d$ respectively.) However, this does not imply directly that their sum is selfadjoint; to prove that it is the idea must be borrowed of examining the inverse of $I+\bar{d} d^{*}+\bar{\delta} \delta^{*}$. That this operator is one-to-one follows from the (semi-boundedness) inequality

$$
\left(\left[I+\bar{d} d^{*}+\bar{\delta} \delta^{*}\right] \alpha, \alpha\right)=(\alpha, \alpha)+\left(d^{*} \alpha, d^{*} \alpha\right)+\left(\delta^{*} \alpha, \delta^{*} \alpha\right) \geqq(\alpha, \alpha) .
$$

For the inverse we shall establish the identity

$$
\left(I+\bar{d} d^{*}+\bar{\delta} \delta^{*}\right)^{-1}=\left(I+\bar{d} d^{*}\right)^{-1}+\left(I+\bar{\delta} \delta^{*}\right)^{-1}-I \equiv S .
$$

By the theorem cited, $S$ is a bounded self-adjoint operator defined on all of $H^{p}$; therefore $S^{-1}$ is also self-adjoint.

The selection of $S$ as the value of the inverse can be motivated. Let $A=\bar{d} d^{*}$ and $B=\bar{\delta} \delta^{*}$. In view of the adjointness of $d$ and $\delta$, one has $d^{*}$ an extension of $\delta$ and $\delta^{*}$ an extension of $d$. Formally, $A$ and $B$ can be multiplied in either order to give $O$ (actually $A B C O$ and $B A \subset O$; see below), and

$$
\begin{aligned}
\frac{I}{I+A+B} & =\frac{I}{I+A+B+A B}=\frac{I}{(I+A)(I+B)} \\
& =\frac{I}{I+A}+\frac{I}{I+B}-I .
\end{aligned}
$$

The proof of (1) begins with the observation that $\delta^{*} \bar{d} \subset O$ (i.e. $\delta^{*} \bar{d}$ is a contraction of the operator $O$ ) and has as domain precisely the domain of $\bar{d}$. For suppose $\alpha$ in the domain of $\bar{d}$ : then there is a sequence of $C^{2}$ forms $\left\langle\alpha^{i}\right\rangle$ approaching $\alpha$ such that $\left\langle d \alpha^{i}\right\rangle$ approaches $\bar{d} \alpha$. Therefore

$$
(\delta \theta, \bar{d} \alpha)=\lim \left(\delta \theta, d \alpha_{i}\right)=\lim \left(\theta, d d \alpha_{i}\right)=0
$$

for all $\theta$ in the domain of $\delta$; that is, $\delta^{*}(\bar{d} \alpha)=0$. The corresponding result is valid for $d^{*} \bar{\delta}$; an immediate consequence of these two facts is that $A B=0$ on the domain of $B$, and $B A=0$ on the domain of $A$.

It will now be shown that $(I+A+B) S=I$. Begin by observing that since $(I+B)^{-1}$ is defined on all of $H^{p}$ (see above),

$$
(I+B)^{-1}-I=(I-[I+B])(I+B)^{-1}=-B(I+B)^{-1},
$$


and similarly

$$
(I+A)^{-1}-I=-A(I+A)^{-1} .
$$

These two facts imply that

$$
\begin{aligned}
A S & =A(I+A)^{-1}+A\left[(I+B)^{-1}-I\right] \\
& =I-(I+A)^{-1}-A B(I+B)^{-1}=I-(I+A)^{-1} .
\end{aligned}
$$

Similarly,

$$
B S=I-(I+B)^{-1} \text {. }
$$

Consequently,

$$
\begin{aligned}
(I+A+B) S= & (I+A)^{-1}+(I+B)^{-1}-I+I \\
& -(I+A)^{-1}+I-(I+B)^{-1}=I .
\end{aligned}
$$

Since, as was already noted, $I+A+B$ is one-to-one, this equation reveals that it is precisely $S^{-1}$; therefore $I+A+B$ is self-adjoint and maps onto $H^{p}$.

It is now possible to show that $\bar{\Delta}$ itself is self-adjoint (and therefore equal to its extension $\left.\bar{d} d^{*}+\bar{\delta} \delta^{*}\right)$. For $\Delta_{1}=\Delta+I$ is a contraction of $\bar{d} d^{*}+\bar{\delta} \delta^{*}+I$. Therefore $\Delta_{1}$ is one-to-one and the inverse mapping is bounded; furthermore, $\left(\bar{\Delta}_{1}\right)^{-1}$ is defined on a closed set. To show that this closed set is all of $H^{p}$ it will suffice to prove that every $C^{1}$ form $\gamma$ in $H^{p}$ (the $C^{1}$ forms constitute a dense subset of $H^{p}$ ) is in the range of $\Delta_{1}$. Whether $\gamma$ is $C^{1}$ or not, there is a form $\alpha$ such that $\left(\bar{d} d^{*}+\bar{\delta} \delta^{*}+I\right) \alpha=\gamma$, as has just been seen.

Consider $(\Delta \theta, \alpha)$ : since $\alpha$ is in the domain of $\bar{d} d^{*}+\bar{\delta} \delta^{*}$ one has

$$
(\Delta \theta, \alpha)=\left(\delta \theta, d^{*} \alpha\right)+\left(d \theta, \delta^{*} \alpha\right)=\left(\theta,\left[\bar{d} d^{*}+\bar{\delta} \delta^{*}\right] \alpha\right)=(\theta, \gamma-\alpha) .
$$

By the fundamental differentiability lemma ( $\$ 3)$ it follows that $\alpha$ is $C^{2}$. If one forms $\delta \alpha$ pointwise, then on every compact set $N$ the form $\delta \alpha-d^{*} \alpha$ is orthogonal to the dense class of $C^{1}$ forms whose carriers are contained in the interior of $N$. Therefore $\delta \alpha=d^{*} \alpha$ everywhere, and similarly $d \alpha=\delta^{*} \alpha$; consequently $\left(\bar{d} d^{*}+\bar{\delta} \delta^{*}\right) \alpha$ reduces to $\Delta \alpha$. Therefore $\Delta_{1} \alpha=\gamma$, the range of $\Delta_{1}$ is dense, and so $\bar{\Delta}_{1}=\bar{d} d^{*}+\bar{\delta} \delta^{*}+I$. Since the latter operator was seen above to be self-adjoint, so is $\bar{\Delta}_{1}$ and with it $\bar{\Delta}=\bar{\Delta}_{1}-I$.

TheOREM. On an orientable Riemannian manifold with $C^{k}$ Riemann tensor $(k=5, \cdots, \infty)$ and negligible boundary, the closure of $\Delta$ is self-adjoint.

If $d$ and $\delta$ had been restricted to forms of class $C^{m}, 1 \leqq m \leqq k-5$, the same proof could have been carried through. The closure of $\Delta$ is thus independent of the value of $m$.

By our original method (see $[12 ; 13]$ ), using the Friedrichs mollifier, we obtained the self-adjointness of $\bar{\Delta}$ when $k=2$.

One use of the self-adjointness of $\bar{\Delta}$ should be the extension of the heat 
equation method of Milgram and Rosenbloom [19] to open manifolds with negligible boundary. By the spectral theorem $\bar{\Delta}=\int \lambda d E_{\lambda}$; one would define $W_{t}=\int e^{-t \lambda} d E_{\lambda}$ and hope to derive the properties of $W_{t}$ directly from this representation( $(5)$.

Since $\bar{\Delta}$ is self-adjoint, $\Delta^{-1}$ is densely defined on $F^{\perp}$, the orthogonal complement of the space of harmonic forms. (For an element orthogonal to the range of a self-adjoint operator is in its null space.) Define $G$ as in $\$ 1$. One can show that

$$
\bar{d} \bar{\Delta} \subset \bar{\Delta} \bar{d}
$$

(i.e. $\bar{d} \bar{\Delta}$ is a contraction of $\bar{\Delta} \bar{d}$ ) and use this to show that on the domain of $\bar{d}$

$$
\bar{d} G \subset G \bar{d} \quad \text { (on domain of } \bar{d} \text { ). }
$$

In particular, $\bar{d} G=G \bar{d}$ whenever both are defined. On the other hand, if $G$ is a bounded operator, as it is for compact manifolds, $\bar{d} G$ will also be a bounded operator defined on all of $H^{p}$ and will satisfy $G \bar{d} \subset \bar{d} G$.

3. The fundamental differentiability lemma $\left({ }^{6}\right)$. In this section we shall drop our previous convention and use $\Delta$ to denote the pointwise operator with no norm restrictions. We wish to prove the

Fundamental differentiability lemma. Let $M$ be an orientable Riemannian manifold with Riemann tensor of class $C^{k}, k=5, \cdots, \infty$;

Let $\alpha$ be a measurable p-form such that $\int \alpha * \alpha$ is finite over every compact subset of $M$;

Let $\beta=\gamma+\lambda \alpha$, where $\gamma$ is $a C^{m} p$-form, $1 \leqq m \leqq k-5$ (when $k=5$ take $\left.m=1\right)$, and $\lambda$ is a real number;

Assume that $(\Delta \theta, \alpha)=(\theta, \beta)$ for every $C^{k-2} p$-form $\theta$ with compact carrier;

Then $\alpha$ is (equal almost everywhere to) $a C^{m+1}$ form and $\Delta \alpha=\beta$.

See [7], [18], and [23].

If for the moment $\Delta$ is viewed as a linear transformation with domain restricted to compact carried $C^{k-2}$ forms, the last hypothesis can be stated:

$$
\Delta^{*} \alpha=\beta .
$$

The conclusion $\Delta \alpha=\beta$ will follow easily when $\alpha$ is known to be $C^{2}$. For then $(\Delta \theta, \alpha)=(\theta, \Delta \alpha)$, and since $(\Delta \theta, \alpha)$ also equals $(\theta, \beta)$, one obtains $(\theta, \Delta \alpha-\beta)=0$ for all compact carried $C^{2}$ forms $\theta$. But the $C^{k-2}$ forms vanishing outside a fixed compact set $K$ form a dense subset of the corresponding Hilbert space of $p$-forms on $K$, so that $\Delta \alpha-\beta=0$ almost everywhere on $K$. Therefore $\Delta \alpha-\beta=0$ almost everywhere on $M$.

Assume that $k \geqq 6$ (in the appendix a very brief outline is given of the modifications necessary when $k=5$ ). The standard ([1], [7], and [16])

(5) Added in proof. This has now been carried out; see [25].

(6) We are indebted to Professor K. Kodaira for his helpful suggestions. 
Kneser parametrix is the double $p$-form

$$
\omega(y, x)=\frac{\sum \rho(y, x) r^{2-n}(y, x)}{p !(n-2) n v_{n}} A_{I, J}(y, x) d y^{I} d x^{J}
$$

whose detailed definition is given in the appendix. Here we remark that on a general open manifold $\omega$ can be defined only on a compact subset, which for us is a neighborhood of an arbitrary point $P ; y$ is restricted to the neighborhood $S$ (see appendix) of $P$ throughout.

The differentiability of $\alpha$ will follow from the relation

$$
\alpha(y)=(\omega[y, x], \beta[x])-\left(\Delta_{x} \omega[y, x], \alpha[x]\right) ;
$$

our task is to obtain (2) without assuming that $\alpha$ is differentiable (cf. [1]).

Following Kodaira [18, pp. 621-622] (see also Courant-Hilbert, vol. II, p. 228) we modify $\omega$ to $\omega^{\epsilon}(\epsilon>0)$ by replacing $r^{2-n}$ with $F^{\epsilon}(r)$, where

$$
\begin{array}{ll}
F^{\epsilon}(r)=\frac{1}{2 \epsilon^{n}}\left[n \epsilon^{2}-(n-2) r^{2}\right] \equiv A-B r^{2} & \text { for } r \leqq \epsilon, \\
F(r)=r^{2-n} & \text { for } r \geqq \epsilon .
\end{array}
$$

[F€ $(r)$ is obtained by taking the tangent at $r^{2}=\epsilon^{2}$ to $r^{2-n}$, viewed as a function of $r^{2}$.] $\omega^{\epsilon}(y, x)$ is $C^{k-2}$ [same as $\omega(y, x)$ ] except when $r=\epsilon$, where it is $C^{1}$. Were it not for this behavior at $r=\epsilon$ we would clearly have

$$
\left(\Delta \omega^{\epsilon}, \alpha\right)=\left(\omega^{\epsilon}, \beta\right),
$$

and in fact $\left(2^{\prime}\right)$ is true despite the behavior. This is verified by a patching argument as follows:

Define the $C^{\infty}$ functions

$$
\begin{aligned}
& f(t)=0 \text { for } t \leqq-1, \quad f(t)=1 \text { for } t \geqq 0 ; \\
& 0 \leqq f(t) \leqq 1 \text { for }-1 \leqq t \leqq 0 ; \\
& F^{e, \delta}(r)=A-B r^{2}+f\left(\frac{r-\epsilon}{\delta}\right)\left[r^{2-n}-\left(A-B r^{2}\right)\right](\epsilon>\delta>0) .
\end{aligned}
$$

Use the latter in place of $F^{\epsilon}(r)$ to define $\omega^{\epsilon, \delta}$. Since $F^{\epsilon, \delta}(r)$ is $C^{\infty}$ in $r$ we certainly have

$$
\left(\Delta \omega^{\epsilon, \delta}, \alpha\right)=\left(\omega^{\epsilon, \delta}, \beta\right) .
$$

On the other hand, $\omega^{\epsilon, \delta}=\omega^{\epsilon}$ except in the shell $\epsilon-\delta<r<\epsilon$, so it will suffice to show that as $\delta$ approaches 0 the contribution of this shell to $\left(2^{\prime \prime}\right)$ approaches 0 . On the right this is clear enough, but on the left the situation is complicated by derivatives up to the second order. The key in treating these derivatives is (cf. Friedrichs $[10$, p. 141]) that the various terms which arise, for example 


$$
\frac{1}{\delta^{2}} f^{\prime \prime}\left(\frac{r-\epsilon}{\delta}\right) \frac{\partial r}{\partial x^{i}} \frac{\partial r}{\partial x^{j}}\left[\frac{1}{r^{n-2}}-\left(A-B r^{2}\right)\right],
$$

have bounds independent of $\delta$. For since $r^{2-n}$ and $A-B r^{2}$ agree at $r=\epsilon$ along with their first derivatives, $r^{2-n}-\left(A-B r^{2}\right)$ is $\leqq C \delta^{2}$ in the shell. If we compute $\Delta \omega^{e, \delta}$ and use this device and its obvious modifications we see that the function being integrated on the shell is bounded. Since the volume of the shell approaches $0,\left(2^{\prime \prime}\right)$ yields $\left(2^{\prime}\right)$ in the limit.

Let $T=T(y, \epsilon)$ be the sphere of radius $\epsilon$ about $y .\left(2^{\prime}\right)$ can be rewritten

$$
(\Delta \omega, \alpha)-(\Delta \omega, \alpha)_{T}+\left(\Delta \omega^{\epsilon}, \alpha\right)_{T}=(\omega, \beta)-(\omega, \beta)_{T}+\left(\omega^{\epsilon}, \beta\right)_{T} .
$$

Take the limit as $\epsilon$ goes to zero: $(\Delta \omega, \alpha)_{T},(\omega, \beta)_{T}$, and $\left(\omega^{\epsilon}, \beta\right)_{T}$, which are all functions of $y$, converge to zero in the $L_{2}$ norm on $S$. It suffices to show this for the individual components, which in all three cases are sums of terms of the form

$$
f_{\epsilon}(y)=\int V_{I, J}(y, x) D^{J}(x)(g(x))^{1 / 2} d x=\int V(y, x) D(x)(g(x))^{1 / 2} d x,
$$

where $V(y, x)$ is $O\left(r^{1-n}\right)$ uniformly in $y$ [see appendix; actually $\Delta \omega$ is $O\left(r^{2-n}\right)$ ], vanishing when $x$ is not near $y$, and $D(x)$ is square integrable (properties which are independent of the Riemann tensor). Let

$$
U=|V| \text { and } E=\left|D g^{1 / 2}\right|,
$$

and let $C$ be a bound on $\int U(y, x) d x$, independent of $y$.

$$
\begin{aligned}
\left|f_{\epsilon}(y)\right| & \leqq \int(U(y, x))^{1 / 2}\left[(U(y, x))^{1 / 2} E(x)\right] d x \\
\left|f_{\epsilon}(y)\right|^{2} & \leqq \int U(y, x) d x \int U(y, x) E^{2}(x) d x \leqq C \int U(y, x) E^{2}(x) d x, \\
\int_{S}\left|f_{\epsilon}(y)\right|^{2} d y & \leqq C \int_{x}\left[\int_{y} U(y, x) d y\right] E^{2}(x) d x \leqq C^{2} \int_{T} E^{2}(x) d x .
\end{aligned}
$$

Since $D^{2}(x)$ is integrable, $\int_{T} D^{2}(x) d x$ goes to zero with $\epsilon$, taking with it $\int_{S}\left|f_{\epsilon}(y)\right|^{2} d y$. Note that it has been established that $V(y, x)$ is the kernel of a bounded Hilbert space operator.

$\left(\Delta \omega^{\epsilon}, \alpha\right)_{T}$ behaves differently and provides us with the "punch out" term-i.e., approaches $\alpha(y)$. We prove this first with $\alpha$ replaced by a continuous form $k(y)=\sum^{\prime} K_{I}(y) d y^{I}$. It is shown in the appendix that

$$
\Delta_{x} \omega^{e}(y, x)=n(n-2) \epsilon^{-n} \eta+\frac{n}{2} \epsilon^{2-n} \Delta \eta+\epsilon^{-n} O(r),
$$

where $\eta=r^{n-2} \omega$. (The lead term is made plausible by the fact that in euclidean 
space $\Delta r^{2}=-2 n$.) Working with the lead term, we have

$$
\begin{aligned}
n(n-2) v_{n} \int_{T} \eta(y, x) * \kappa(x) & \\
= & \sum_{I}^{\prime}\left[\int_{T} \sum_{J} \rho(y, x) A_{I, J}(y, x) K^{J}(x)(g(x))^{1 / 2} d x\right] d y^{I} .
\end{aligned}
$$

An elementary calculation (using the basic properties of normal coordinates) shows that $A_{i, j}(y, y)=g_{i j}(y)$, so as $\epsilon$ approaches zero $\rho(y, x) A_{I, J}(y, x) K^{J}(x)$ uniformly approaches $G_{I, J}(y) K^{J}(y)=K_{I}(y)$. But $\int_{T}(g(x))^{1 / 2} d x=v_{n}+O(\epsilon)$, so $n(n-2) \epsilon^{-n} \int_{T} \eta(y, x) * \kappa(x)$ approaches $\kappa(y)$. The other terms in $\Delta \omega^{e}$ do not contribute to the limit, and $\left(\Delta \omega^{\epsilon}, \kappa\right)_{T}$ converges uniformly to $\kappa(y)$.

The general case is now easy if we combine the special case just treated with the fact that (by the same proof as above) $\Delta \omega^{\mathbf{e}}$ is the kernel of a bounded integral operator; let the bound be $C^{\prime}$. Given a positive $\delta$ choose a continuous $\kappa$ such that

$$
L_{2}(\alpha-\kappa)<\delta / 2\left(C^{\prime}+I\right),
$$

and then choose $\epsilon_{0}$ so small that $\epsilon<\epsilon_{0}$ implies that the norm of

$$
\left(\Delta \omega^{\epsilon}, \alpha\right)_{T}-\alpha=\left(\Delta \omega^{\epsilon}, \alpha-\kappa\right)_{T}+\left[\left(\Delta \omega^{e}, \kappa\right)_{T}-\kappa\right]+\kappa-\alpha
$$

is less than $\delta$. (See Kodaira [18, p. 626]; also compare Friedrichs [10, pp. 527$528]$.) Thus we see that $\left(2^{\prime}\right)$ yields (2) as a limiting case almost everywhere in $S$.

After a few preliminaries the differentiability of $\alpha$ is now obtained by differentiating under the integral sign; see below.

Appendix. We shall follow the outline given by de Rham [7] to establish the properties of the parametrix $\omega(y, x)$, at the same time examining $\omega^{e}$. We then show that equation (2) of $\$ 3$ implies the fundamental lemma.

Fix a coordinate system about an arbitrary point $P$, and select a positive $s$ so small that in the sphere $S^{*}$ of radius $2 s$ centered at $P$ the normal coordinate functions $z^{i}(y, x)$ (see below) exist and are of class $C^{k-1}$; and, in addition, that if $y$ is fixed in $S^{*}$ and $z^{i}(y, x)$ used as coordinates, then normal coordinates $v\left(z, z^{\prime}\right)$ exist and are $C^{k-2}$ in $S^{*}$. Let $S$ be the sphere of radius $s$; $S^{\prime}$ the sphere of radius $s / n$.

Construct a $C^{\infty}$ function $\bar{\rho}(t)$ whose extreme values are 0 and 1 and which satisfies

$$
\bar{\rho}(t)=1 \text { when } t \leqq s / 4 n ; \quad \bar{\rho}(t)=0 \text { when } t \geqq s / 2 n .
$$

Define

$$
\rho(y, x)=\bar{\rho}(r[y, x]) .
$$

The constant $v_{n}$ is the volume of the unit sphere in euclidean $n$-space. 
Define $A_{i, j}(y, x) \equiv-\partial^{2} r^{2}(y, x) / \partial y^{i} \partial x^{j}$. By the conventions for capital indices, $\sum A_{I, J} d y^{I} d x^{J}$ is then defined (it is equal to $\sum^{\prime} p$ ! [Det $\left.A(I, J)\right] d y^{I} d x^{J}$ ). For $y$ in $S$ define

$$
\omega(y, x) \equiv \sum \frac{\rho(y, x) r^{2-n}(y, x)}{p ! n(n-2) v_{n}} A_{I, J}(y, x) d y^{I} d x^{J} .
$$

(We shall omit the minor changes necessary when $n=2$.) $\omega(y, x)$ is invariant.

The distance $r(y, x)$ is given (when small) by the formula

$$
r^{2}(y, x)=\sum g_{i j}(y) z^{i} z^{j}=\sum \bar{g}_{i j}(z) z^{i} z^{i} .
$$

Here $z^{i}(y, x)$ are the normal coordinates of $x$ issuing from $y$, and the bar on $\bar{g}_{i j}$ indicates that the components are given with respect to the $z$ coordinate system. For a concise summary of normal coordinates as well as an exposition of material used below see Bochner [2].

We wish to establish the "parametrix property": $\Delta_{x} \omega(y, x)$ is $O\left(r^{2-n}\right)$. The first step is to compute $\Delta\left(r^{2-n}\right)$ (see Feller [8, p. 639]); concurrently we compute $\Delta r^{2}$. For functions, $\Delta$ is the Laplace-Beltrami operator which is given coordinate-wise by

$$
\Delta=-\sum \frac{1}{\bar{g}^{1 / 2}} \frac{\partial}{\partial z^{i}}\left[\bar{g}^{1 / 2} \bar{g}^{i j} \frac{\partial}{\partial z^{j}}\right] .
$$

$\partial r^{2} / \partial z^{j}=2 \sum g_{j m}(x) z^{m}=$ (property of normal coords.) $2 \sum \bar{g}_{j m}(z) z^{m}$, and therefore

$$
\sum \bar{g}^{i j}(z) \frac{\partial r^{2}}{\partial z^{j}}=2 \sum \delta_{m}^{i} z^{m}=2 z^{i}
$$

Consequently

$$
\begin{aligned}
\Delta\left(r^{2-n}\right) & =\Delta\left(r^{2}\right)^{(2-n) / 2}=\sum(n-2) \frac{1}{\bar{g}^{1 / 2}} \frac{\partial}{\partial z^{i}}\left[\bar{g}^{1 / 2} r^{-n} z^{i}\right], \\
\Delta r^{2} & =\sum-\frac{2}{\bar{g}^{1 / 2}} \frac{\partial}{\partial z^{i}}\left[\bar{g}^{1 / 2} z^{i}\right] .
\end{aligned}
$$

But

$$
\sum \frac{\partial}{\partial z^{i}}\left[\left(r^{2}\right)^{-n / 2} z^{i}\right]=0
$$

while

$$
\sum \frac{\partial}{\partial z^{i}}\left(z^{i}\right)=n,
$$


so

$$
\begin{aligned}
\Delta\left(r^{2-n}\right) & =\left(\frac{n-2}{2}\right) \frac{1}{\bar{g}(z)} r^{-n} \sum z^{i} \frac{\partial \bar{g}(z)}{\partial z^{i}}, \\
\Delta r^{2}+2 n & =-\frac{2}{\bar{g}(z)} \sum z^{i} \frac{\partial \bar{g}(z)}{\partial z^{i}} .
\end{aligned}
$$

Because $\partial \bar{g}(z) / \partial z^{i}$ is zero at $z=0$, the right-hand side of the last equation and the factor of $r^{-n}$ above it are both $O\left(r^{2}\right)$, uniformly in $y$ near $P$.

This result can be used in the study of $\Delta \omega$ in view of the following product formula established by de $\operatorname{Rham~([7,~p.~53];~cf.~Kodaira~[18,~p.~612]),~an~}$ invariant formula resulting from his explicit evaluation of $\Delta$ :

$$
\Delta(f \eta)=f(\Delta \eta)-2 \nu+(\Delta f) \eta
$$

where $\nu=\sum^{\prime} N_{K} d x^{K}$ is defined by

$$
N_{K}=\sum_{i, j} g^{i j} \frac{\partial f}{\partial x^{i}} H_{I, j}
$$

The covariant derivatives of the components of $\eta$ are given by

$$
H_{I, j}=\frac{\partial B_{I}}{\partial x^{j}}-\sum_{v=1}^{p} \sum_{a} B_{i_{1} \cdots i_{v-1} a i_{v+1} \cdots i_{p}} \Gamma_{i_{v} j}{ }^{a} .
$$

Take $f$ to be $r^{2-n}$ and $\eta$ to be $r^{n-2} \omega$. We have just seen that $\Delta f$ is $O\left(r^{2-n}\right)$, as of course is $f$ itself. Therefore the terms $f \Delta \eta$ and $(\Delta f) \eta$ are both $O\left(r^{2-n}\right)$. We have left to show that $\nu$ is $O\left(r^{2-n}\right)$, or equivalently that $H_{I, j}$ is $O(r)$.

Select normal coordinates issuing from a point $Q$ and let $v$ and $u$ be two points with coordinates given in this system. We need the fact (true in any coordinate system) that

$$
\frac{\partial r^{2}(v, u)}{\partial v^{i}}=-2 g_{i m}(v) z^{m}(v, u)
$$

(see de Rham [7, p. 59]). (Note the implication that $r^{2}$ is $C^{k}$. Set $v=0$ (i.e. $v$ is the origin $Q$ ) and differentiate with respect to $u^{i}$. Since $z^{m}(0, u)=u^{m}$ we obtain

$$
\frac{\partial^{2} r^{2}(0, u)}{\partial u^{i} \partial v^{i}}=-2 g_{i j}(0), \text { or } A_{i, j}(0, u)=g_{i j}(0) .
$$

Therefore $\partial H_{I} / \partial u^{i}=0$. On the other hand, $\Gamma_{b c}^{a}(u)$ vanishes at $u=0$ (origin of normal coordinates) and so is $O(r)$. Therefore $\Delta \omega$ is $O\left(r^{2-n}\right)$. (Since at $v=0$ $g_{i j}(v)$ is $g_{i j}(y)$ and $d y^{i}=d v^{i}$ we have shown that $\sum A_{I, J}(y, x) d y^{I} d x^{J}$ $=\sum G_{I, J}(y) d y^{I} d u^{J}$ with components independent of $u$.) We also need-what is readily verified - that the first partial derivatives 
of the components of $\Delta \omega$ are $O\left(r^{1-n}\right)$.

Returning to the product formula for $\Delta$ and using the determination of $\Delta r^{2}$, we see that setting $f=r^{2}$ and again $\eta=r^{n-2} \omega$ yields

$$
\Delta \omega^{\epsilon}(y, x)=n(n-2) \epsilon^{-n} \eta+\frac{n}{2} \epsilon^{2-n} \Delta \eta+\epsilon^{-n} O(r) ;
$$

we don't need the sharper $O\left(r^{2}\right)$.

We now have the tools to derive the differentiability of $\alpha$ from the equation

$$
\alpha(y)=\int \omega(y, x) * \gamma(x)+\int\left[\lambda \omega(y, x)-\Delta_{x} \omega(y, x)\right] * \alpha(x),
$$

where (in order to have room for iteration) we restrict $y$ to the open sphere $S^{\prime}$. The first integral-as is seen below-gives a $C^{m+1}$ form in $y$; it is the second which occupies our attention. We utilize the paper of Bochner [2, Lemmas 1, 2, 4, and 5]. (The components on the right are integrals of the type he considers.) Briefly, by iterating (1) until the kernel is in $L_{2}$ it follows that $\alpha(y)$ is bounded (i.e. each $A_{I}$ or, equivalently, $|\alpha|$ bounded). When $\alpha(x)$ is known to be bounded, (1) yields the continuity of $\alpha(y)$; when $\alpha(x)$ is continuous, (1) implies that $\alpha(y)$ is $C^{1}$, and the derivatives are obtained by differentiation under the integral sign.

We now digress on an elementary fact. The normal coordinates $z^{i}(y, x)$ satisfy $\bar{g}_{i j}(0)=g_{i j}(y)$; we need normal coordinates $w^{i}(y, x)$ such that the corresponding tensor satisfies $\tilde{g}_{i j}(0)=\delta_{i j}^{j}$. Let $G$ be the matrix of $g_{i j}(y)$. The positive definite quadratic form $X G X^{\prime}$ can be written as a sum of squares by a linear transformation. Indeed, set

$$
Y_{1}=g_{11}^{-1 / 2}\left[g_{11} X_{1}+g_{12} X_{2}+\cdots+g_{1 n} X_{n}\right]
$$

and proceed by induction. If $Y=X M$, then $M^{-1} G\left(M^{-1}\right)^{\prime}=I$.

Take normal coordinates $z^{i}(y, x)$ and then introduce the coordinates $w^{i}(y, x)$ by $w(y, x)=z(y, x) M(y)$.

We follow Kodaira in using the coordinates $w^{i}$ as the independent variables in the integrands. We wish to show that $\alpha$ is $C^{L}(1 \leqq L \leqq m)$ implies that $\alpha$ is $C^{L+1}$; we illustrate with the integral

$$
\int w^{i} \frac{\partial(\tilde{g}(w))^{1 / 2}}{\partial w^{i}} r^{-n} B_{I, J}(y, x) A^{J}(x) d w .
$$

Begin by taking $\partial^{L} / \partial y^{L}$ ( $w$ fixed) underneath the integral sign, bearing in mind that $x=x(y, w)$. Since $r^{2}=\sum\left(w^{i}\right)^{2}, r^{-n}$ is unscathed, as is $w^{i}$.

$$
\frac{\partial(\tilde{g}(w))^{1 / 2}}{\partial w^{i}}
$$


requires special comment, since $\tilde{g}$ is a function of $y$ as well as $w$. But

$$
\frac{\partial \tilde{g}(0)}{\partial w^{i}} \equiv 0 \quad \text { so } \quad \frac{\partial^{L+1}}{\partial y^{L} \partial w^{i}}(\tilde{g}(w))^{1 / 2}
$$

has a zero at $w=0$. In view of this, if we now switch the variable of integration back to $x$ we can use the cited Lemma 2 to take one more derivative. Applying the same method to the other terms, we obtain by induction the fundamental lemma.

When $k=5$ the parametrix $\omega$ must be changed to the noninvariant $\omega^{5}$ by using the original coordinate system in $S$ and replacing $A_{I, J}(y, x)$ by $G_{I, J}(y)$. This economizes on differentiability, and equation (1) is still valid. But $\omega^{5}$ is only $O\left(r^{1-n}\right)$, the difficulty being that $H_{I, J}$ is no longer $O(r)$.

By iterating equation (1) once the poles can be made $O\left(r^{2-n}\right)$, but we must also show that the first derivatives are $O\left(r^{1-n}\right)$. This is done with the aid of the function

$$
\phi\left[\frac{r^{2}(y, x)}{r^{2}(y, z)}\right],
$$

where the $C^{\infty}$ function $\phi$ is 1 for $t \leqq 1 / 3$ and 0 for $t \geqq 2 / 3$. From this one obtains $\alpha(y)$ is $C^{1}$. With the aid of the coordinates $w^{i}$ and the continued use of this iteration method a second derivative can be obtained.

\section{BIBLIOGRAPHY}

1. P. Bidal and G. de Rham, Les formes differentielles harmoniques, Comment. Math. Helv. vol. 19 (1946) pp. 1-49.

2. S. Bochner, Analytic mapping of compact Riemann spaces into euclidean space, Duke Math. J. vol. 3 (1937) pp. 339-354.

3. A. B. Brown, Critical curvatures in Riemannian spaces, Duke Math. J. vol. 3 (1937) p. 475.

4. C. Chevalley, Theory of Lie groups, Princeton, 1946, Chaps. III and V.

5. G. de Rham, Sur l'analysis situs des varietes a $n$ dimensions, J. Math. Pures Appl. (1931) pp. 115-200.

6. - Sur la theorie des formes differentielles harmoniques, Annales de l'Université de Grenoble vol. 22 (1946) pp. 135-152.

7. G. de Rham and K. Kodaira, Harmonic integrals, Institute for Advanced Study, Mimeographed Notes, 1950.

8. W. Feller, Lösungen der linearen partiallen Differentialgleichungen zweiter Ordnung vom elliptischen Typus, Math. Ann. vol. 102 (1930) pp. 633-649; p. 639, footnote.

9. K. O. Friedrichs, On differential operators in Hilbert spaces, Amer. J. Math. vol. 61 (1939) pp. 523-544.

10. - The identity of weak and strong extensions of differential operators, Trans. Amer. Soc. vol. 55 (1944) pp. 132-151.

11. - On the boundary-value problems of the theory of elasticity and Korn's inequality, Ann. of Math. vol. 48 (1947) pp. 441-471.

12. M. Gaffney, The harmonic operator for exterior differential forms, Proc. Nat. Acad. Sci. U.S.A. vol. 37 (1951) pp. 48-50. 
13. - The harmonic operator for exterior differential forms, dissertation, University of Chicago, March, 1951.

14. - A special Stokes's theorem for complete Riemannian manifolds, Ann. of Math. vol. 60 (1954) pp. 140-145.

15. W. V. D. Hodge, A Dirichlet problem for harmonic functionals, with applications to analytic varieties, Proc. London Math. Soc. (2) vol. 36 (1932) pp. 257-303. 1941.

16. - The theory and applications of harmonic integrals, Cambridge University Press,

17. K. Kodaira, Über die harmonischen Tensorfelder in Riemannschen Mannigfaltigkeiten, Proc. Imp. Acad. Tokyo vol. 20 (1944) pp. 186-198, 257-261, 353-358.

18. - Harmonic fields in Riemannian manifolds (generalized potential theory), Ann. of Math. vol. 50 (1949). pp. 587-665.

19. A. Milgram and P. Rosenbloom, Harmonic forms and heat conduction I: closed Riemannian manifolds, Proc. Nat. Acad. Sci. U.S.A. vol. 37 (1951) pp. 180-184.

20. B. Sz. Nagy, Spektraldarstellung linearer Transformationen des Hilbertschen Raumes, Berlin, 1942.

21. F. Rellich, Ein Satz ibber mittlere Konvergenz, Nachr. Ges. Wiss. Göttingen (1930) pp. 30-35.

22. A. Weil, Sur les théorèmes de de Rham, Comment. Math. Helv. vol. 26 (1952) pp. 119145.

23. H. Weyl, Method of orthogonal projection in potential theory, Duke Math. J. vol 7 (1940) pp. 411-444.

24. - On Hodge's theory of harmonic integrals, Ann. of Math. vol. 44 (1943) pp. 1-6.

25. Added in proof. M. Gaffney, The heat equation method of Milgram and Rosenbloom for open Riemannian manifolds, Ann. of Math. vol. 60 (1954) pp. 458-466.

26. Added in proof. K. Yosida, An ergodic theorem associated with harmonic integrals, Proc. Imp. Acad. Tokyo vol. 27 (1951) pp. 540-543.

University of Chicago,

Chicago, Ill.

Princeton University,

Princeton, N. J. 